\title{
Correction to: Comparing Acute Kidney Injury Reports Among Antibiotics: A Pharmacovigilance Study of the FDA Adverse Event Reporting System (FAERS)
}

\author{
Taylor M. Patek ${ }^{1,2} \cdot$ Chengwen Teng $^{1,2}$ (D) Kaitlin E. Kennedy ${ }^{1,2} \cdot$ Carlos A. Alvarez $^{3} \cdot$ Christopher R. Frei $^{1,2,4,5}$
}

Published online: 26 June 2020

(c) Springer Nature Switzerland AG 2020

\section{Correction to: Drug Safety (2020) 43:17-22 \\ https://doi.org/10.1007/s40264-019-00873-8}

In the original publication of the article, the second sentence in the figure 2 caption should read as below:

A total of 2,042,801 reports (including 20,138 AKI reports) were included in the study. This is an example demonstrating how to calculate RORs: AKI RORs for vancomycin plus cefepime $=[22 /(113-22) /((20,138-22) /(2,042,80$ $1-20,138-(113-22)))]=24.31$.

The original article can be found online at https://doi.org/10.1007/ s40264-019-00873-8.

Chengwen Teng

tengc3@livemail.uthscsa.edu

1 Pharmacotherapy Division, College of Pharmacy, The University of Texas at Austin, San Antonio, TX, USA

2 Pharmacotherapy Education and Research Center, Long School of Medicine, The University of Texas Health Science Center at San Antonio, 7703 Floyd Curl Dr., MSC-6220, San Antonio, TX 78229, USA

3 Division of Clinical/Translational Research, Hodge School of Pharmacy, Texas Tech University Health Sciences Center, Dallas, TX, USA

4 South Texas Veterans Health Care System, San Antonio, TX, USA

5 University Health System, San Antonio, TX, USA 